\title{
A new Joint Radio Resource Management scheme in heterogeneous wireless networks based on handover
}

\author{
Abbas Mirzaei Somarin ${ }^{1, *}$, Mehdi NosRATI ${ }^{2}$, Morteza BARARI ${ }^{3}$, Human ZARRABI ${ }^{4}$ \\ ${ }^{1}$ Department of Computer Engineering, Ardabil Branch, Islamic Azad University, Ardabil, Iran \\ ${ }^{2}$ Department of Computer Engineering, payame noor university (pnu) iran p.o Box, 19395-3697 Tehran, \\ Iran \\ ${ }^{3}$ Faculty of Information, Communications and Security Technology, Malek Ashtar University of \\ Technology, Tehran, Iran \\ ${ }^{4}$ Integrated Network Management Group, ICT Research Institute (ITRC) Tehran, Iran
}

\begin{abstract}
We have introduced a new management method in this paper named as the adaptive JRRM which selects the best RAT through considering various parameters such as the type of the service, information about the location of the user and the user's movement and the service cost. The suggested resources of the analytical model based on the Petri networks have been provided for the Joint Radio Resource Management method and have been implemented and evaluated in the SPNP environment. The obtained numerical results indicate that the adaptive JRRM method reduces the rate of vertical transitions between RATs which will provide more stable connections for the users. The results also indicate that the proposed method provides less service cost for the operators and this can be highly regared in implementing heterogeneous wireless networks.
\end{abstract}

Keywords: Joint Radio Resource Management, heterogeneous wireless networks, selecting a wireless access technology, vertical handoff

\section{Introduction}

The development of wireless and mobile technologies, have made a revolution in the world of communications. These technologies are in progress towards access to broadband information from among several network platforms to provide comprehensive availability between the programs and services. Broadband availability systems include wireless local area networks, wireless metropolitan area networks and wireless personal area networks. In addition, frequently-used mobile access technologies such as GPRS, WCDMA, EDGE, third generation (3G) communications systems, WiMAX and Bluetooth are in this category.

In order to provide the requested multimedia services and the quality of the desired service for the mobile users, the Radio Access Technologies are integrated and create a heterogeneous wireless network. Such a network is composed of multiple wireless networks and creates the fourth generation or the next generation of wireless networks. Heterogeneous wireless access is the broad support of IP-based traffic and the best support of mobility are the main motivations for architecturing such a network [1]. 
One of the main isssues in HWN networks is the optimal use of radio resourcs. Although there are many approaches for the management of radio resources in each network, these approaches are not appropriate for the wireless networks. Therefore, a new concept has been created as Joint Radio Resource Management.

Selection methods of RAT which include RAT selection algorithms at the time of starting the meeting and also RAT selection at the time of transferring communications under conduction from a RAT to another RAT (vertical handoff), is the main element of radio resorce management methods at heterogeneous wireless networks. There have been several methods provided for selecting RAT in articles which have investigated different criteria based on a purpose they have. In some papers, the issue of load balance and the network stability has been investigated [3-5]. In some others, the descisions have been made based on the type of the demanded service [6-8]. In some others, such as functionoriented [9] and math-oriented methods [10] which have been decided through several criteria, the purpose is the high efficiency of the network and increasing the users' satisfaction. In some methods, we will notice unnecessary vertical handoffs in the network due to a policy used in them, and this factor has led to the unsustainability of the services and an increase in costs. In previous works to which we referred, the users' movement and his future position in the process of descision making has been considered. Considering this information in the descision making process, will highly prevent the unnecessary vertical handoffs which are created due to the mobility of the user. We aim to provide the user with a new method for JRRM which decreases vertical handoffs in the network and the sustainability of the services through the new information.

Although the issue of seleting RAT has been investigated in numerous articles, but the presented strategies has often been investigated based on the simulation at the system level and little attention has been given to providing analytical methods. In this thesis, the proposed method has been evaluated based on the analytical model of random reward networks.

In the end, in the second chapter, we will deal with investigating heterogeneous wireless networks and the reasons for their emergence as well as the challenges in these networks. In the third chapter, we will deal with the classification and investigating resource management methods at HWN networks. Then, in the fourth chapter, we will first deal with introducing random reward networks and the works conducted in this area. Then, in the fourth chapter, we will introduce and explain the JRRM managemet method proposed by the adaptive JRRM. Then, in the fifth chapter, we will investigate the proposed method through the analytical model of random reward network and will compare it to methods raised in HWN networks, such as service-based and load balancing. Finally, in the sixth chapter, after summing up the results, we will provide recommendations for future works.

In wireless communications, there are various networks with Radio access standards and Technologies with capabilities and limitations of their own. We can generally divide 
wireless networks into two categories: heterogeneous and homogeneous. When in an environment where the wireless networks are used all the networks are of equal standards and technology, they are called as "homogeneous wireless networks". The present approach in fourth generation wireless networks is the use of the capabilities of different networks with different standards and technologies together which raise a new concept called "heterogeneous wireless networks". As shown in Figure 1 each of these access networks have different efficiency and coverage and may overlap other networks as well.

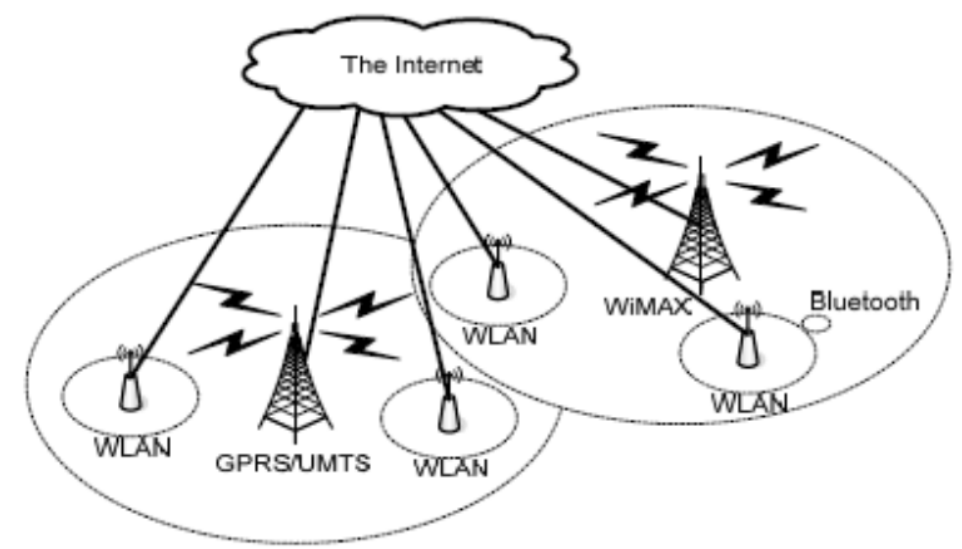

Figure 1: Overlaping the covered areas in a heterogeneous wireless networks

Old and present RATs, along with today's new technologies, create a new concept as Always Best Connected (ABC) [11]. ABC means that the user receives service from the most appropriate RAT, in a seamless manner and in accordance with service requirements and according to the predetermined criteria. The concept of $\mathrm{ABC}$ has been indicated in Figure 2-2 through a simple example [12]. In Figure 2, in area (a) the user starts a new session in the house environment and for the first time the WLAN network is selected for his desired service. When the user leaves home to go to his office, as the WLAN network can no longer hold communication, the rest of the customer service is seamlessly transferred to a $3 G$ network (point i). In area (b), the mobile users will benefit from radio resource management by $3 G$ network. Finally, in the area (c) when the user is located in the WLAN network range of his company (point ii) the user's connection is transferred from the 3G network to WLAN.

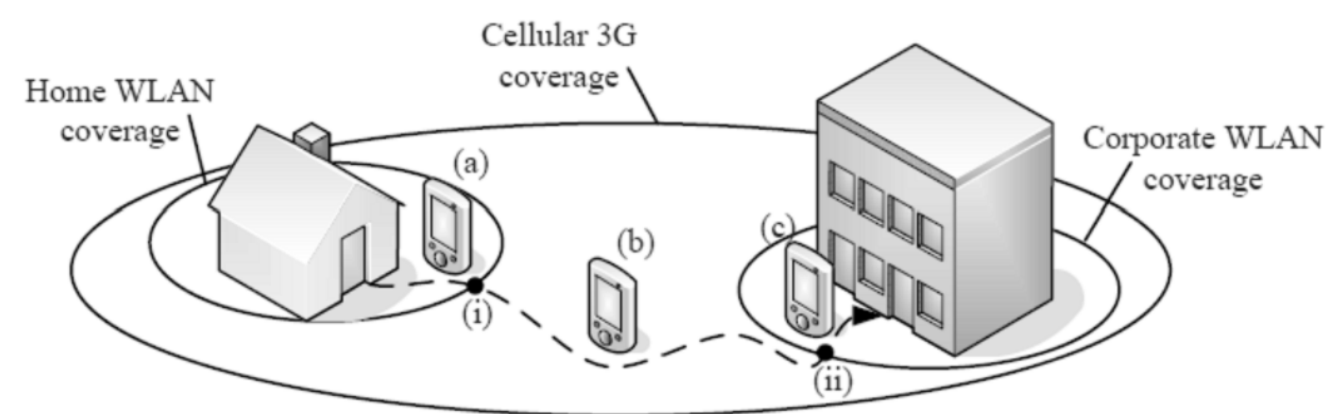

Figure 2: An example of user mobility and the concept of ABC 


\section{Mobility and mobility management}

In wireless networks, the users are mobile and can freely move from one place to another. The users can have access to networks at any place and any time. Portability for users enables them to navigate among network devices, session migrations, and finally get similar services or better than the previous services in the new network. The difference of a mobile device with a portable device is that portable devices are disconnected when moving, whereas mobile devices maintain their connection with the network while moving. Therefore different requirements must be considered for mobile users, so that its connection will be maintained during mobility. Therefore, we first provide a precise definition of the concept of "mobility". There are different types of mobility. Some common examples of mobility are: mobility of the users, data (including flows), software (including agents, users) or hardware (terminals) [3].

Terminal mobility refers to the time when a device changes position form an access network to another access network. Terminal mobility enables the users to move in the network, while maintaining their connection and availability. This mobility is divided into two types of "macro-mobility" and "micro-mobility". "Micro-mobility" refers to the mobility within a domain and "macro-mobility" refers to the mobility between the domains. Domain refers to one or a combination of several subnets which are known as the autonomous system. The protocols used in each domain may be different from the other. Micro-mobility is usually managed by wireless access technology and in data link layer. Macro-mobility includes mobility between the domains, either domains (subnets) with the similar technology, or between domains with different technology. Macromobility often brings changes in IP address.

Flow mobility means as the displacement of flows moving from an intermediate or terminal to another. The movement can take place between an access technology and a similar or dissimilar terminal.

Session mobility is a kind of displacement where all the flows relevant to a session are moving simultaneously, and user mobility is a kind of mobility that all flows are moved to a new terminal. Session mobility is often managed at the transport or application layer. For example, Session Initiation Protocol manages the sessions at the application layer and the Stream Control Transmission Protocol provides support in the transport layer.

There are discussions about the most appropriate level for mobility management. Mobility management approaches exist in all layers of the network protocol that relate to different types of movements. Real-time applications may not be able to tolerate delivery delay, packet loss, etc. and may prefer to manage mobility to adapt to changes. Non-realtime applications may not manage mobility themselves and may need support from the network layer. Both applications should be able to work together. 


\section{Radio resource management in wireless access networks}

Radio Resource Unit is defined as a set of physical transmission parameters needed to support transmission of user data in communication networks. Physical transmission parameters depend on the techniques used for multiple accesses.

For example, in FDMA, a certain bandwidth that is inside a carrier frequency is considered as RRU. In TDMA, a pair of carrier frequency and time slot has been considered as RRU. In CDMA, the carrier frequency, code order and power level is considered as RRU. The main difference that exists in CDMA is that the power level required to support user interaction is not a fixed value, but depends on the level of interference. Therefore, transmission power resources will vary over time according to a number of factors such as propagation conditions, interference, load of the cells and etc. [16].

"Radio Resource Management" includes strategies, algorithms, and mechanisms to control radio transmission parameters in wireless communication systems to effectively use the limited resources allocated to network [17].

\section{Radio resource management in heterogeneous networks: Joint Radio Resource Management}

Realizing the ABC concept in a heterogeneous network scenario where different RATs have been placed together, suggests a new concept of RRM that must take into account all available resources in different RATs and consider the resources as similar, therefore, JRRM strategies and methods occur [2].

Since each RAT is based on unique multiple access mechanisms (such as FDMA, TDMA, CDMA), radio resource management in such networks has its own issues. Local RRM mechanisms mentioned in the previous section is required in order to make effective use of radio resources available in each RAT (Figure 3.(a)). In addition, JRRM is responsible for the management of radio resources in the system and considers the available radio resources in each RAT as a resource pool and manages them in a synchronized way (Figure 3.(b)). 


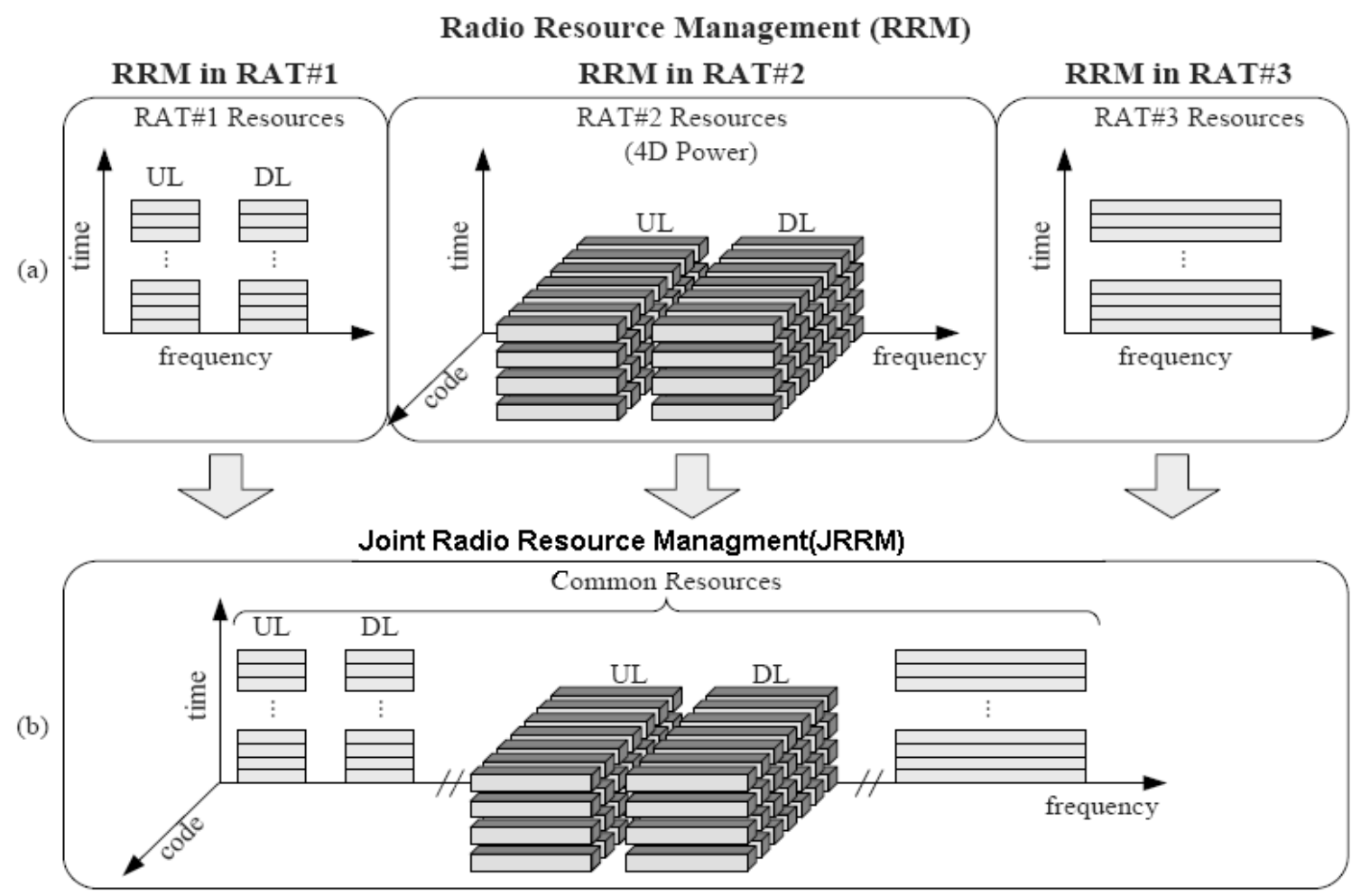

Figure 3: Radio resource management. (a) RRM independently for each RAT. (b) Joint RMM on a pool of resources

As mentioned in the previous section, RRM functions which arise in the field of an independent RAT, is the admission control, congestion control, traffic scheduling, horizontal handoff (handoff inside the system) and so on. When these functions in heterogeneous networks scenario act harmoniously with each other between different RATs, they include the concept of Joint, so that these methods consider the information of several RATs for making decisions. As a result, the function can be referred to as Joint admission control, Joint Congestion Control, Joint Traffic Scheduling and so on.

When the scenario of heterogeneous networks is considered, a new challenge arises as RAT selection procedure. This procedure is used in order to select the most suitable RAT for a service requested at the time of the session (which is known as initial RAT selection) and during a running session (which is known as the vertical handoff (VHO)).

Returning to the simple example in Figure 2.2, this procedure is explained. As indicated in the Figure, in stage (a), the selection procedure RAT, selects home WLAN (in order to improve the bit rate) for the user. When the user gets out of the WLAN coverage area (point i), the vertical handoff mechanisms are set up to transfer the user's session to a 3G network without any interruption in the service. When the user gets into the company's WLAN range (point ii), the network decides to st another vertical handoff in order to convey a session to the WLAN.

It should be noted that selecting RAT has been known as one of the key elements in JRRM methods, in order to achieve maximum benefit of the flexibility offered by different RATs in heterogeneous networks. RAT selection procedure is conducted by 
taking into account various criteria (such as type of service, load conditions on the network, etc.) to achieve the ultimate objective of improving total capacity, optimal use of resources and quality of service.

\section{Interaction between RRM and JRRM entities: Interactions between RRM and JRRM entities include two main functions.}

1) Information reporting function which allows the RRM to report relevant information to the JRRM unit that controls it. This can also be done periodically and implemented through the occurrence of an event. Information also includes information of fixed cells (cell communications, capabilities and capacities of the cells, the quality of cellular service, the highest bit rate available for any given service, the average buffer delay and etc.) as well as the dynamic data (the amount of cell load, the cell's sent and received ability, interference of the cell, etc.) [2]. These teo functions are also used in exchanging information between different entities of JRRM. 2) Another function which exists is the RRM Decision Support function, which specifies the interaction between the RRM and JRRM at the time of the decision. There are two methods of decision-making in these interactions. 1) JRRM is at the center of decision-making so that the final decisions is made by this unit and in order to carry out these decisions, makes RRM entities aware of them. 2) In the second method, the local RRM is at the center of decision-making, so that the JRRM entity will only provide recommendations to the RRM entities and the final decision will be made by the local RRM. Figure 4 indicates the interaction model of JRRM.

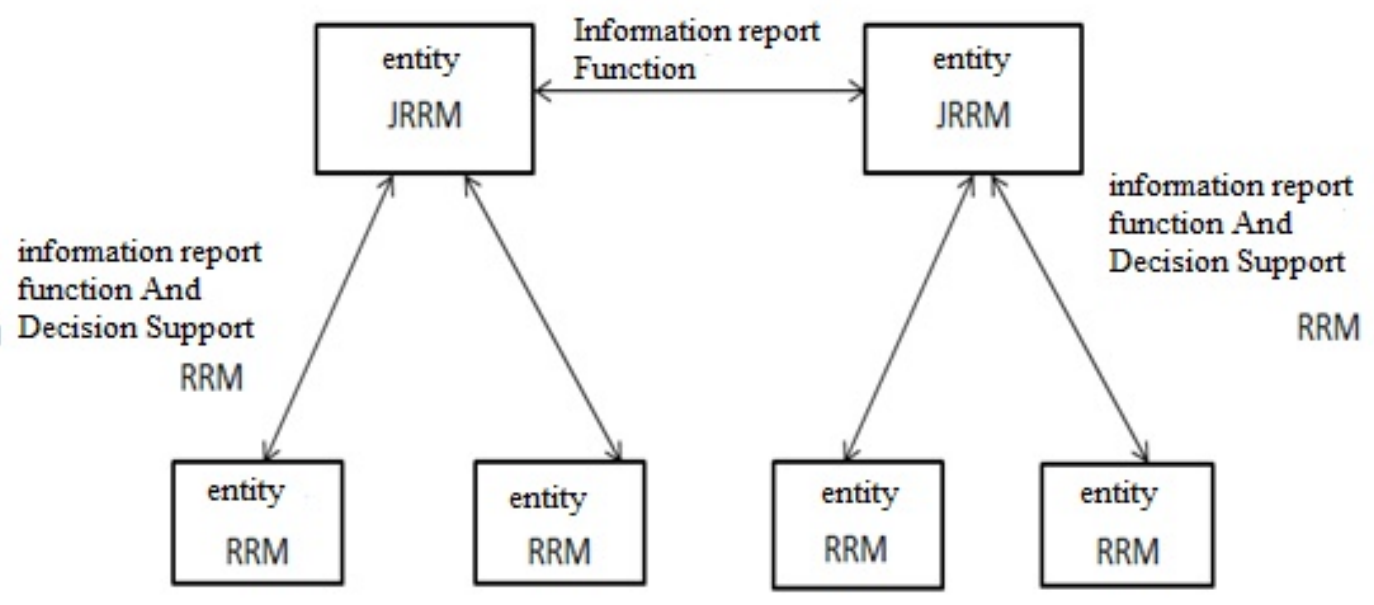

Figure 4: Iinteraction between JRMM and RRM

Based on different performances of local RRM and JRRM entities, another type of interaction arises between them. In [3] four degrees of interactions have been proposed which are summarized in the following table. As shown in the table, in a high degree of interactions, more functions have been transferred from the local RRM entity to the JRRM entity. Stronger interactions between RRM and JRRM entities are followed by better management of radio resources, whereas this issue leads to an increased network overhead and the signaling rate due to the highly necessary interactions between the 
entities. As indicated in Table 1 in high levels of interactions, decisions are made in a shorter time scale. Now, in most studies we focus on low to medium levels of interactions.

Table 1: The degree of interaction between the RRM and JRRM entities

\begin{tabular}{|c|c|c|c|}
\hline Existent functions in RRM & $\begin{array}{c}\text { Existent functions in } \\
\text { JRRM }\end{array}$ & $\begin{array}{l}\text { Interaction } \\
\text { Time scale }\end{array}$ & $\begin{array}{c}\text { Interaction } \\
\text { degree }\end{array}$ \\
\hline $\begin{array}{l}\text { - The initial RAT } \\
\text { selection } \\
\text { - VHO } \\
\text { - Admission control } \\
\text { - congestion control } \\
\text { - HHO } \\
\text { - traffic scheduling } \\
\text { - power control }\end{array}$ & $\begin{array}{l}\text { - Configuration and } \\
\text { utilization of } \\
\text { politics }\end{array}$ & Day/ hour & little \\
\hline $\begin{array}{l}\text {-, Admission control } \\
\text { - congestion control } \\
\text { - HHO } \\
\text { - traffic scheduling } \\
\text { - power control }\end{array}$ & $\begin{array}{l}\text { - Configuration and } \\
\text { utilization of politics } \\
\text { - The initial } \\
\text { RAT selection } \\
\text { - VHO }\end{array}$ & Minute & medium \\
\hline $\begin{array}{ll}\text { - } & \text { traffic scheduling } \\
\text { - } & \text { power control }\end{array}$ & $\begin{array}{ll}\text { - } & \text { Configuration and } \\
& \text { utilization of } \\
& \text { politics } \\
\text { - } & \text { VHO } \\
\text { - } & \text { The initial RAT } \\
& \text { selection } \\
\text { - } & \text { Admission control } \\
\text { - } & \text { congestion control } \\
\text { - } & \text { HHO } \\
\end{array}$ & second & more \\
\hline - power control & $\begin{array}{ll}\text { - } & \text { Configuration and } \\
& \text { utilization of } \\
& \text { politics } \\
\text { - } & \text { VHO } \\
\text { - } & \text { The initial RAT } \\
& \text { selection } \\
\text { - } & \text { Admission control } \\
\text { - } & \text { congestion control } \\
\text { - } & \text { HHO } \\
\text { - } & \text { Traffic schedulling }\end{array}$ & ms & Very much \\
\hline
\end{tabular}

User service

Type of service is one of the important parameters in JRRM algorithms. Here, two types of services are taken into account, real-time and non- real-time services. Because the real-time services are delay-sensitive and require better service quality in comparison to the non-real-time service, they must be allocated to a service which will provide better and more stable relationship for them and the conduction of VHO process in real-time services must be avoided due to delays in the VHO process which causes instability of the service. But it is not so in real-time services because these services are not timesensitive. In any case, unnecessary vertical handoffs for real-time and non-real-time 
services are undesirable because of the delay they create and lead to the loss of communication.

Information related to the mobility type and predicting the position of the user

At the intended area of HWN, the user can be in two positions, the user is either under the cover of CDMA 2000 and can only have access to CDMA 2000 technology, or is located in the hot spot area and has access to both RATs (CDMA 2000 and IEEE 802.11.

The user is known as the hot-spot user, if the received signal strength from IEEE 802.11 becomes greater than or equal to the default threshold value, the user will be known as out of hot-spot user.

In [17], a method has been provided that can provide the future position of the user for a good release channel and it has been indicated that under good conditions, the accuracy of this method is more than $80 \%$. Therefore, the proposed method in [17] can be used to predict the position of the user. The procedure to predict the position, makes JRRM aware from the issue that whether the active user (who is now the hot-spot point), will get out of the hot-spot point or will stay in that areaduring his communication. This information prevents unnecessary vertical transitions which will be discussed later.

One of the key parameters used for making decisions, is the speed of the user at the hotspot area. In [19], a method has been presented to estimate the user's speed. Based on a default threshold value for the speed of the user, the users can be divided into both vehicular users (users who have high mobility) and non-vehicular users (users who have low mobility).

JRRM algorithms can make better decisions using these parameters, which will increase quality of service and reduce unnecessary vertical handoffs and reduce operator costs. We will evaluate these algorithms in next sections.

\section{RAT Selection Algorithm}

In this algorithm, JRRM makes necessary decisions to select the appropriate RAT based on the information it has gathered. RAT selection algorithm consists of three steps.

The JRRM entity receives the new call request.

JRRM, requests information related to the position and movement of the user from the location register and location predictor units.

Based on the information collected by the JRRM unit and the rules that follows, the most suitable RAT for the session will be selected.

1) If the user is located out of the hot-spot area, the management of resources will be conducted by the radio resource management unit in CDMA 2000. 
2) Otherwise, for the vehicular user who is in the hot-spot area, CDMA 2000 will be selected for Resource Management to avoid repeated VHO which may occur due to the movement of the vehicular user.

3) For the non-vehicular user who is in the hot-spot area, the service type is considered for selecting RAT.

If the condition (type of service == non-real-time service) is true, IEEE 802.11, will be selected for the user due to high bandwidth and low service cost. Otherwise, if the condition (type of service == real-time service) is true, according to the information of predicting the position of the user, the appropriate RAT will be selected. So that, if it is predicted that the user will get out of the hot-spot area, IEEE 802.11 will be selected for him (to ensure stability of the service), otherwise, CDMA 2000 will be an appropriate choice for the user to avoid unnecessary VHO.

To better clarify the process of the RAT selection algorithm, its flowchart is provided below. (Figure 5)

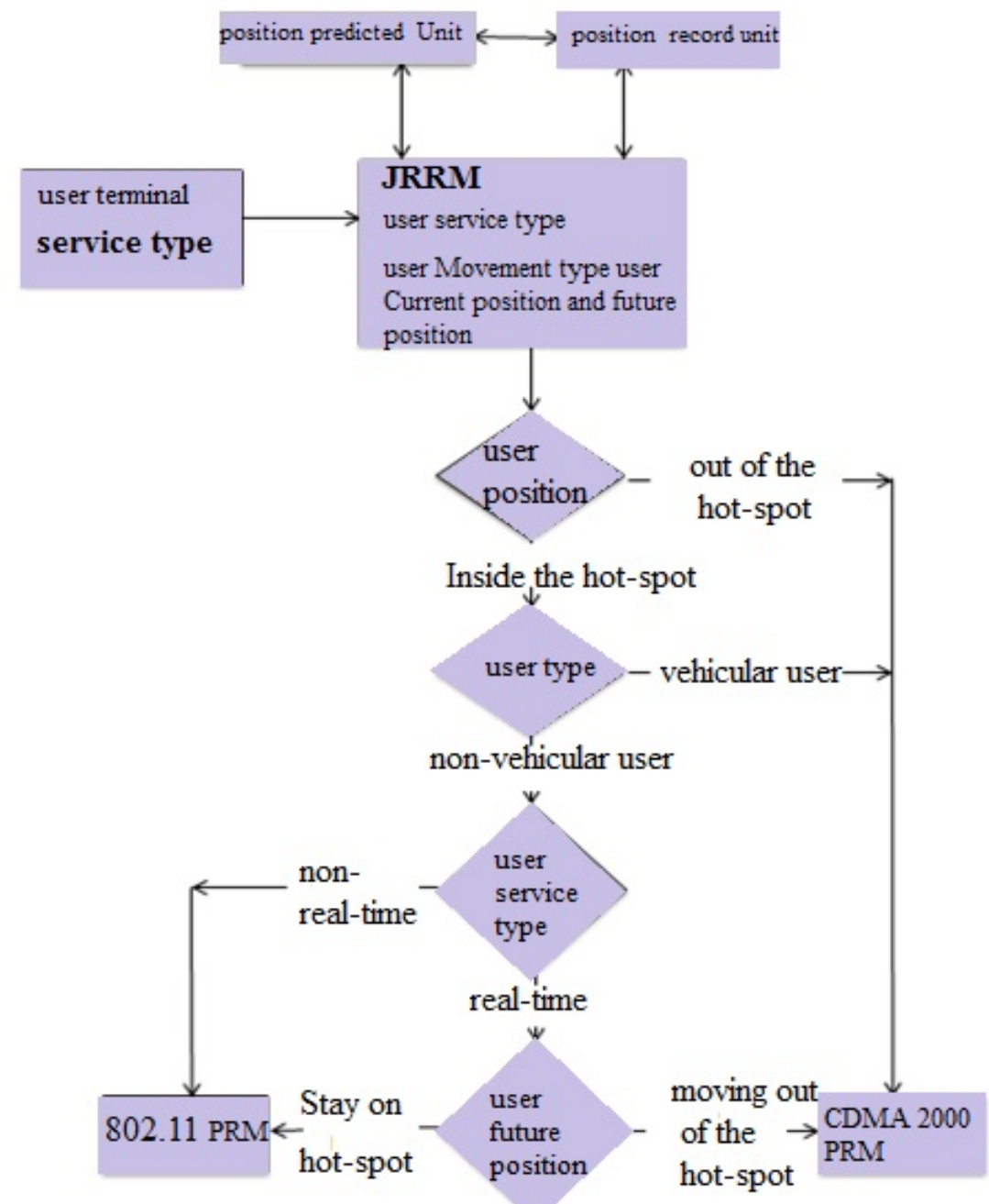

Figure 5: RAT selection algorithm flowchart 


\section{VHO Algorithm}

The vertical handoff process may occur due to the users' mobility, improvement in the quality of service or reduction in service costs. The proposed VHO algorithm consists of two steps.

1) JRRM periodically reviews all sessions under its management to recognize their position changes.

2) If a user changed position, so that was moving out of the hot-spot area and was using the IEEE 802.11 technology, an inter-system handoff occurs from IEEE 802.11 to CDMA 2000 due to loss of signal strength received from IEEE 802.11. But if the user moved into the hot-spot area from the out hot-spot area, the appropriate RAT is selected to conduct the handoff act depending on the user service.

For non-real-time services that belong to a vehicular user, no handoff occurs in order to avoid repeated vertical handoffs, on the contrary, an inter-system occurs from CDMA 2000 to IEEE 802.11 for a non-vehicular user in order to reduce service costs and increase throughput. For time-sensitive real-time services, service stability is more important than the cost of service and throughput. Thus, the vertical handoff decisions must be made with sufficient accuracy. To prevent successive vertical handoffs in a realtime service, the information of predicting the position of the user are applied. So that, if it is predicted that the user will move out of the hot-spot area during his session, no handoff will occur. Otherwise, a vertical handoff to IEEE 802.11 will be conducted.

To better clarify how the VHO algorithm acts, its flowchart is given below. 


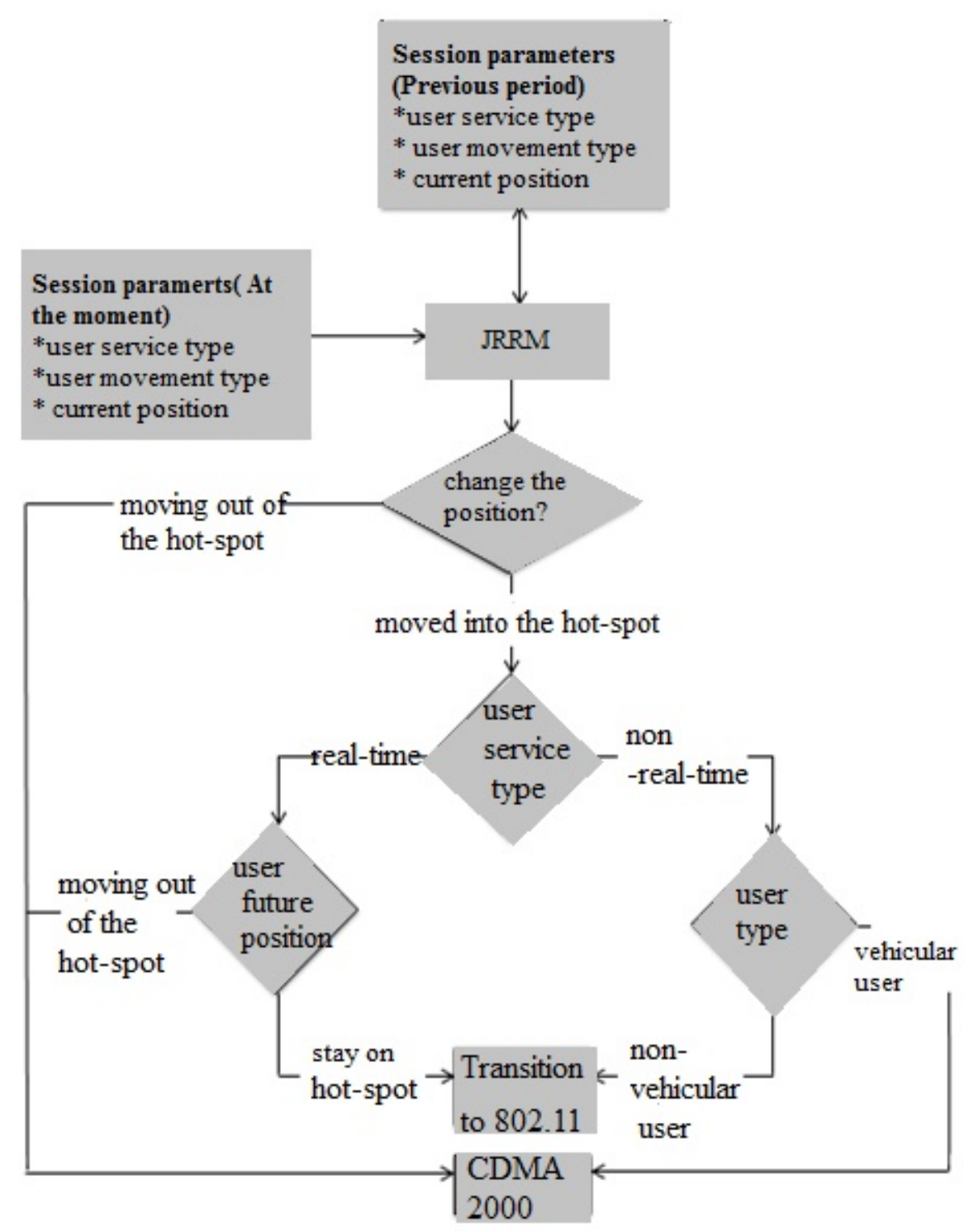

Figure 6: Flowchart of the VHO algorithm procedure

- Customer service model

Service model which is considered, implies the following three assumptions.

The probability that the user is placed in the hot-spot area is characterized as $P_{\text {hotspot }}$ [20]. Therefore, the probability that the user is out of the hot-spot area, is $\left.1-\mathrm{P}_{\text {hotspot}}\right)$ ).

The new requests to the HWN network enter according to a Poisson process with an average arrival rate of $\lambda$ [20]. The admission request is either a real-time or a non-realtime service which is random and is determined with the probability of $\mathrm{P}_{\mathrm{rt}}$ and $\mathrm{P}_{\mathrm{nrt}}$ [8].

$$
\mathrm{P}_{\mathrm{rt}}+\mathrm{P}_{\mathrm{nrt}}=1
$$

The service time of an exponential distribution request is considered with an average $1 / \mu$, where $\mu$ is the average rate of services completion [16]. 
- User mobility model

In this thesis, a probability-oriented mobility model in accordance with [15] has been considered for the users. So that, the probability that the the user will exit of the hot-spot point during its session $\mathrm{P}_{\text {exit }}$, or enter it is considered with a probability of $\mathrm{P}_{\text {enter }}$, and the probability that the user is a vehicular user (with high mobility) or aa non-vehicular user is (with low mobility), have been specified with the probability of $\mathrm{P}_{\mathrm{v}}$ and $\mathrm{P}_{\mathrm{nv}}$, respectively.

Considering small quantities for $\mathrm{P}_{\text {exit }}$, specify a hot-spot scenario related to a company where the users will stay for a long time in the hot-spot area (we mean their own company), on the contrary, considering large quantities for $\mathrm{P}_{\text {exit }}$ corresponding to a general area covered by WLAN (such as airports) where users are commuting, is arranged.

Under equilibrium conditions, the average number of users who exit the hot-spot point and the users who enter this area is equal [16].

As a result, we will have:

$$
\begin{array}{r}
P_{\text {exit }} \times P_{\text {hotspot }}=P_{\text {enter }} \times\left(1-P_{\text {hotspot }}\right) \\
P_{\text {enter }}=\frac{P_{\text {exit }} \times P_{\text {hotspot }}}{\left(1-P_{\text {hotspot }}\right)}
\end{array}
$$

ocation change | vehicular user $)=\frac{P(\text { location change \&\& vehicular user })}{P(\text { vehicular user })}$

$$
=1
$$

$\mathrm{P}_{\text {exit }}$ and $\mathrm{P}_{\text {enter }}$ values have important roles in evaluating $\mathrm{VHO}$ process in HWN environments.

For users of the vehicular type, due to their high speed, it is assumed that the probability that their location changes from the inside the point to the outside of the hot-spot, is 1.

As a result, we will have:

$$
\begin{aligned}
\text { ve } P(\text { location change | nonvehivular user }) \\
=\frac{P(\text { location change \&\& nonvehicular user })}{P(\text { nonvehicular user })} \\
=\frac{P(\text { location change })-P(\text { location change \&\& vehicular user })}{P(\text { nonvehicular user })} \\
=\frac{P(\text { location change })-P(\text { vehicular user })}{P(\text { nonvehicular user })}=\frac{P_{\text {exit }}-P_{v}}{P_{n v}}
\end{aligned}
$$


Using conditional probability and the relationship (5) we can conclude the nonvehicularity of the user and changing position as follows:

The outstanding feature of this method, is taking into account various parameters such as the type of user service, the type of user mobility, the user's current and future position in the decision-making process. Using this information, JRRM can make optimal decisions in order to avoid repeated and unnecessary vertical handoffs that reduce the operators' service cost and this reduction in service cost will be highly considered for the implementation of heterogeneous wireless networks. Another feature of this method is to ensure the quality of customer service and maintaining the sustainability of customer service.

In this paper, we will evaluate the adaptive JRRM method which was introduced in the fourth chapter. For this purpose, we will model the adaptive JRRM method through SRN. In this thesis, SPNP tool has been used for the implementation and evaluation of SRN models. In former section, we will introduce Petri networks and SPNP, in section last we will deal with the details of the analytical model and in section 3-5, after explaining the employed evaluation criteria, we will analyze and evaluate the results obtained from the analysis of the adaptive JRRM in network different conditions and will compare the performance of this method with the methods employed in HWN like the service-based methods and the load-balance-oriented method.

For validating the considered SRN model, we consider states of the system where the results obtained from the presented algorithms are clear. For example, if we consider a user's exit from the hot-spot point as $\left(\mathrm{P}_{\text {exit }}\right) 0.1$ - it means that the mobility of the users is very low and the users who enter the hot-spot area, will less likely exit this area during their service - and for the state where the staying probability of the users in the hot-spot area $\left(\mathrm{P}_{\text {hotspot }}\right)$ is zero - it means that no user inside the hot-spot requests for service according to the RAT selection algorithm which was introduced in introduction, all the system load must enter into CDMA 2000 network and through the gradual increase of $\mathrm{P}_{\text {hotspot, }}$ the network load on CDMA 2000 decreases and increased on IEEE 802.11. Because according to the RAT selection algorithm, for the non-vehicular users who are in the hot-spot area $\left(\mathrm{P}_{\mathrm{nv}}=0.9\right)$ due to low mobility conditions $\left(\mathrm{P}_{\text {exit }}=0.1\right)$, the IEEE 802.11 network will be selected. Thus, much of the network load enters to the IEEE 802.11 RAT and a small part of the load will go to the CDMA 2000 network. As indicated in Figure 7-5, the results of SRN model for users' probability to stay in the hot spot area are consistent with these results.

\section{Conclusion}

In this paper we have introduced a new management method in this paper named as the adaptive JRRM which selects the best RAT through considering various parameters such as the type of the service, information about the location of the user and the user's movement and the service cost. The suggested resources of the analytical model based on the Petri networks have been provided for the Joint Radio Resource Management method and have been implemented and evaluated in the SPNP environment. The obtained 
numerical results indicate that the adaptive JRRM method reduces the rate of vertical transitions between RATs which will provide more stable connections for the users. The results also indicate that the proposed method provides less service cost for the operators and this can be highly regared in implementing heterogeneous wireless networks.

\section{References}

[1] E. Hossain. Heterogeneous Wireless Access Networks: Architectures and Protocols, Springer, 2008

[2] L. Giupponi, R. Agusti, J. Perez-Romero and O. Sallent. "Joint Radio Resource Management Algorithm for Multi-RAT Networks,” IEEE Global Telecommunications Conference, GLOBECOM '05, pp. 3850-3855, Dec. 2005.

[3] 3GPP, "Improvement of RRM across RNS and RNS/BSS (post rel-5) (release 6),” TR 25.891 v0.3.0, 3rd Generation Partnership Project (3GPP), 2003.

[4] D. Niyato and E. Hossain. "Wlc04-5: Bandwidth allocation in 4g heterogeneous wireless access networks: A noncooperative game theoretical approach," IEEE Global Telecommunications Conference, GLOBECOM '06, pp. 1-5, Nov. 2006.

[5] A. Tolli, P. Hakalin, "Adaptive load balancing between multiple cell layers," VTC 2002-Fall. 2002 IEEE 56th Vol. 3, pp. 1691-1695, Sept. 2002.

[6] K. Murray, R. Mathur, D. Pesch, "Network access and handover control in heterogeneous wireless networks for smart space environments, " in: First International Workshop on Management of Ubiquitous Communications and Services, MUCS, Waterford, Ireland, Dec. 2003.

W. Zhang, "Performance of real-time and data traffic in heterogeneous overlay wireless networks, " in: Proceedings of the 19th International Teletraffic Congress (ITC 19), Beijing, 2005.

[8] E. Vanem, S. Svaet and F. Paint. "Effects of multiple access alternatives in heterogeneous wireless networks," in Proc. IEEE WCNC, pp. 1696-1700, 2003.

[9] J. P. Romero, O. Sallent, R. Agusti, and M. A. Diaz-Guerra, Radio Resource Management Strategies in UMTS: John Wiley \& Sons, 2005.

[10] Xu Yang, J. Bigham, and L. Cuthbert. "Resource management for service providers in heterogeneous wireless networks," Wireless Communications and Networking Conference, IEEE, Vol. 3, pp. 1305-1310, Mar. 2005.

[11] E. Gustafsson and A. Jonsson. "Always best connected," Wireless Communications, IEEE[see also IEEE Personal Communications], vol. 10, no. 1, pp. 49-55,Feb. 2003.

[12] X. Gelabert. "Contribution to Radio Resource and Spectrum Management Strategies in Wireless Access Networks: A Markov Modeling Approach," Doctoral Thesis, Universitat Politecnica de Catalunya (UPC), Mar. 2010.

[13] R. Brännström. "Mobility Management in Heterogeneous Access Networks," Doctoral Thesis, Lulea University of Technology Sweden, Dec. 2007.

[14] F. IdrisKhan and E. Huh. "An adaptive resource management for mobile terminals on vertical handoff,” Annals of Telecommunications, pp. 435-447, May. 2008. 
[15] K. Piamrat, C.Viho, A. Ksentini and J.M. Bonnin. "Resource Management in Mobile Heterogeneous Networks: State of the Art and Challenges," inria00258507, v 4-3, Mar. 2008.

[16] L. Saker, S Ben. Jemaa and S.E. Elayoubi. "Q-learning for joint access decision in heterogeneous networks," Wireless Communications and Networking Conference, pp. 1-5, Apr. 2009.

[17] X. Gelabert, J. Pérez-Romero, O. Sallent, R. Agustí and F. Casadevall. "Radio Resource Management in Heterogeneous Networks,” 2005.

[18] J. Zander and S. Kim. Radio Resource Management for Wireless Networks, Artech House Publishers: 2001.

[19] M. H. Ahmed, "Call admission control in wireless networks: a comprehensive survey," Communications Surveys \& Tutorials, IEEE, vol. 7, no. 1, pp. 49-68, May. 2005.

[20] L. L. H. Andrew, S. V. Hanly, and R. G. Mukhtar, "Active queue management for fair resource allocation in wireless networks," Mobile Computing, IEEE Transactions on, vol.7, no. 2, pp. 231-246, Dec. 2007. 